
\title{
25 Research Square \\ Silencing Circ_0058063 Enhances Proliferation and Inhibits Apoptosis in PCOS Ovarian Granulosa Cells
}

Chengcai Wen

Huaian Second People's Hospital

Li Zhang ( $\sim$ lizhang201506@aliyun.com )

Huaian Second People's Hospital https://orcid.org/0000-0001-8048-409X

Research

Keywords: circ_0058063, PCOS, ovarian granulosa cells

Posted Date: May 19th, 2020

DOI: https://doi.org/10.21203/rs.3.rs-28740/v1

License: (c) (i) This work is licensed under a Creative Commons Attribution 4.0 International License.

Read Full License 


\section{Abstract}

Background Polycystic ovary syndrome (PCOS) is the most common endocrine disease in reproductiveaged women. This study was designed to explore the role of circ_0058063 in PCOS.

Methods We recruited 25 PCOS patients and 25 no-PCOS patients. The concentrations of follicle stimulating hormone $(F S H)$, testosterone $(\mathrm{T})$, luteinizing hormone $(\mathrm{LH})$, progesterone $(\mathrm{P} 4)$ and estradiol (E2) were measured by radioimmunoassay. The level of aromatase was detected using an ELISA kit. The proliferation and apoptosis of ovarian granulosa cells were assessed using CCK-8 assay and flow cytometry, respectively. Gene and protein expression were evaluated through RT-qPCR and Western blotting assay.

Results The circ_0058063 level in ovarian granulosa cells and follicular fluid is significantly higher in the PCOS group than the no-PCOS group. Besides, silencing circ_0058063 increases the levels of Aromatase mRNA, P4 and E2 in PCOS ovarian granulosa cells. Additionally, silencing circ_0058063 can promote the proliferation of ovarian granulosa cells in patients with PCOS. Furthermore, silencing circ_0058063 can suppress apoptosis of PCOS ovarian granulosa cells.

Conclusions Silencing circ_0058063 enhances proliferation and inhibits apoptosis in PCOS ovarian granulosa cells. Such findings may offer vital insights into a therapeutic target for PCOS.

\section{Introduction}

Polycystic ovary syndrome (PCOS) is the most common endocrine disease in reproductive-aged women $[1,2]$, affecting approximately $10 \%$ of women of childbearing age [3]. The most prominent characteristics of PCOS are abnormal follicular development and hyperandrogenism [4, 5]. Women with PCOS have short-term and long-term complications of multiple systems throughout the body, including reproduction (hairy, amenorrhea, anovulation, infertility and pregnancy complications), metabolism (type 2 diabetes, cardiovascular disease, and abnormal lipid metabolism) and mental (negative, anxiety), which have brought a heavy burden on individuals and the whole society [4]. Presently, PCOS cannot be completely cured. Therefore, the deep exploration of the PCOS development mechanism is particularly significant for the treatment of the disease.

Circular RNAs (circRNAs) constitute a kind of non-coding RNAs, which is named for its closed loop structure, lack of 5 'cap ends, and 3'poly A tails $[6,7]$. Recently, several studies suggest that circRNAs are widely involved in the PCOS progression $[8,9]$. Research evidence shows that the expression of hsa_circ_0043533 and hsa_circ_0043532 in the PCOS group increased evidently than that in the nonPCOS group, while the non-PCOS group had a remarkably lower hsa_circ_0097636 expression as compared to the PCOS group [8]. Research evidence shows that circ_0058063 functions as an oncogene in bladder cancer [10]. However, the involvement of circ_0058063 in PCOS is still not well discovered. Our aim is to explore the function of circ_0058063 in patients with PCOS, which may shed new light on the development of PCOS treatment. 


\section{Methods}

\subsection{Study Subjects}

We recruited 25 PCOS patients and 25 patients with no-PCOS under the age of 35 who underwent in vitro fertilization and embryo transfer (IVF-ET) in the Huai'an Second People's Hospital between January 2018 and April 2019. We measured the subjects' weight, height, and then calculated body mass index (BMI). Selection criteria for the PCOS group (refer to Rotterdam criteria [11]):1) rare ovulation and/or anovulation; 2) clinical manifestations of hyperandrogenism and/or biochemical changes; 3 ) polycystic ovary was found by ultrasound examination. Those who met 2 of the above 3 items were enrolled in the PCOS group. The no-PCOS group was subjects with normal ovaries function, no PCOS, no history of ovarian adverse reactions, no endometriosis, and receiving IVF-ET due to male factors. All patients voluntarily participated in this research project and signed informed consent. This study was approved by the Ethics Committee of the Huai'an Second People's Hospital.

\subsection{Collection of serum}

$5 \mathrm{~mL}$ of fasting venous blood was collected on the morning of the third day of the menstrual cycle. Blood samples were centrifuged at $1500 \mathrm{r} / \mathrm{min}$ for $5 \mathrm{~min}$ to draw the upper layer of serum, aliquoted into a $0.5 \mathrm{~mL}$ EP tube, and stored in the refrigerator at $-80^{\circ} \mathrm{C}$ for testing.

\subsection{Acquisition of follicular fluid}

All subjects induced ovulation according to the GnRH-a/FSH/ HMG/HCG protocol. The dosage and time were adjusted based on the individual situation. 36 hours after HCG injection, the follicles of both ovaries were sucked through the posterior vaginal foramen under the guidance of ultrasound. The first tube of clear follicular fluid without blood staining was collected and centrifuged at $400 \mathrm{xg}$ for $10 \mathrm{~min}$ at room temperature. The supernatant was stored at $-80^{\circ} \mathrm{C}$ until detecting.

\subsection{Collection, separation and purification of ovarian granulosa cells}

We collected the discarded follicle puncture fluid after the separation of the ovarian crown complex, centrifuged it at $2000 \mathrm{rpm}$ for $10 \mathrm{~min}$, and then discarded the supernatant. The cells were resuspended in PBS and digested using $0.25 \%$ trypsin solution at $37^{\circ} \mathrm{C}$ for $5 \sim 10 \mathrm{~min}$. After termination of digestion, the cell suspension was centrifuged at $2000 \mathrm{rpm}$ for $10 \mathrm{~min}$ and resuspended in PBS. Next, we added an equal volume of human lymphocyte separation solution (Tianjin Med Pacific Technology, China) to cells. After centrifugation at $2000 \mathrm{rpm}$ for $20 \mathrm{~min}$, the intermediate cell layer was drawn. The cells were washed with DMEM (Gibco, USA) containing 1\% Pen-Strep (Invitrogen, USA) and 10\% FBS, centrifuged at 
$1000 \mathrm{rpm}$ for $10 \mathrm{~min}$, and resuspended with DMEM. Trypan blue staining proved that the cell survival rate was greater than $90 \%$. The cell density was adjusted to $2 \times 10^{5} / \mathrm{mL}$ and fostered in a $37^{\circ} \mathrm{C}, 5 \% \mathrm{CO}_{2}$ incubator. After the cells adhered to the growth, they were rinsed once or twice every $24 \mathrm{~h}$ and then replaced with fresh culture medium to further purify the mixed blood cells and other impurities.

\subsection{Cell transfection}

Ovarian granulosa cells in the PCOS group were randomly divided into three groups: si-negative control (NC) group (transfected with NC-siRNA), si-circ_0058063-1 group (transfected with circ_0058063-1siRNA), si-circ_0058063-2 group (transfected with circ_0058063-2-siRNA). Cells were transfected by Lipofectamine $^{\text {TM }} 2000$ (Invitrogen, USA) reagent according to the manufacturer's instructions.

\subsection{Hormone levels detection}

The concentrations of follicle stimulating hormone (FSH), testosterone $(\mathrm{T})$, luteinizing hormone $(\mathrm{LH})$, progesterone (P4) and estradiol (E2) were measured by radioimmunoassay. The concentration of aromatase was detected using an ELISA kit. The kits were provided by Xiamen Huijia Biological Technology (Xiamen, China).

\subsection{RT-qPCR}

After 24-hour transfection, the culture medium was replaced, and cells were continued to foster for 48 hours. The total RNA was extracted by Trizol (Invitrogen, USA), and the RNA quality was detected and quantified by ultraviolet spectrophotometry. The cDNA was synthesized using PrimeScript ${ }^{\text {TM }}$ RT Master Mix (Takara, China), and qPCR was performed utilizing TB Green® Premix Ex Taq ${ }^{\text {TM }}$ (Takara, China) according to the standard protocol. $\mathrm{U} 6$ and $\beta$-actin were used as internal reference controls. The relative expression of circ_0058063 and Aromatase mRNA was calculated using the $2^{-\Delta \Delta C t}$ method.

\subsection{Western blotting}

The total protein of the cell was extracted using RIPA buffer (Biyuntian, China). The protein concentration was assessed via a BCA kit (Biyuntian, China). Next, $50 \mu$ l protein samples were taken for SDSpolyacrylamide gel electrophoresis to separate the target protein, and the membrane was transferred by the wet transfer method. We sealed the membrane with $5 \%$ skimmed milk powder for 2 hours, washed the membrane 3 times with PBST solution, and added primary antibodies (1:1000, CST, USA), including Cyclin A, Cyclin D1, Bcl-2, Bax, and GAPDH. Primary antibodies were incubated at $4{ }^{\circ} \mathrm{C}$ overnight. Then a secondary antibody $(1: 3000)$ was added and incubated at $37^{\circ} \mathrm{C}$ for 2 hours. The ECL kit obtained from Amersham Biosciences (Piscataway, $\mathrm{NJ}$ ) was applied to visualize the immunocomplexes. 


\subsection{Cell-Counting Kit-8 Assay}

The cell viability was detected using CCK-8 (Biyuntian, China) based on the instructions. In short, after transfection for 48 hours, $10 \mu \mathrm{l}$ CCK8 reagent was added to ovarian granulosa cells in each well. The cells were incubated at $37^{\circ} \mathrm{C}$ for 4 hours. The microplate reader (Bio-Rad, USA) was used to detect the optical density (OD) at a wavelength of $450 \mathrm{~nm}$.

\subsection{Flow cytometry}

Cell apoptosis was measured utilizing the Annexin V-FITC Apoptosis Detection Kit (Abcam, USA) as the manufacturer's protocol. In short, we added pre-chilled 1 xbinding buffer to resuspend the cells, and then added $10 \mu \mathrm{l} \mathrm{PI}$ and $5 \mu \mathrm{l}$ Annexin V-FIT. Cells were stained for $15 \sim 25 \mathrm{~min}$ in the darkroom. Flow cytometry (BD, USA) was used to detect the apoptosis rate.

\subsection{Statistical analysis}

SPSS 21.0 software was utilized for statistical analysis. The data were expressed as mean \pm standard deviation. The differences between multiple groups were compared with one-way ANOVA followed by LSD multiple comparisons. $P<0.05$ was considered statistically significant.

\section{Results}

\subsection{The general characteristics and hormone levels of patients}

Compared with the no-PCOS group, patients' BMI increases significantly in the PCOS group. Additionally, the PCOS group has significantly higher serum LH and T levels than the no-PCOS group. No statistically significant difference was seen in age and FSH levels between two groups in Table 1. 
Table 1

The general characteristics and hormone levels of patients in this study

\begin{tabular}{|lll|}
\hline Parameter & PCOS $(\mathbf{N}=\mathbf{2 5})$ & No-PCOS $(\mathbf{N}=\mathbf{2 5})$ \\
\hline Age (year) & $29.38 \pm 3.23$ & $30.52 \pm 2.98$ \\
\hline BMI $(\mathrm{kg} / \mathrm{m} 2)$ & $24.32 \pm 5.83$ & $20.73 \pm 3.26^{*}$ \\
\hline FSH $(\mathrm{IU} / \mathrm{L})$ & $5.89 \pm 2.27$ & $5.08 \pm 2.62$ \\
\hline $\mathrm{LH}(\mathrm{IU} / \mathrm{L})$ & $11.95 \pm 6.35$ & $3.38 \pm 1.94^{\star}$ \\
\hline $\mathrm{T}(\mu \mathrm{g} / \mathrm{L})$ & $0.84 \pm 0.31$ & $0.47 \pm 0.28^{*}$ \\
\hline $\begin{array}{l}\text { PCOS, polycystic ovary syndrome; BMI, body mass index; FSH, follicle-stimulating hormone; } \mathrm{LH}, \\
\text { luteinizing hormone; T, testosterone. }{ }^{*}, P<0.05\end{array}$ \\
\hline
\end{tabular}

\subsection{The expression of circ_0058063 is elevated in PCOS ovarian granulosa cells and follicular fluid}

As presented in Fig. 1A and 1B, qRT-PCR results indicate that the circ_0058063 level in ovarian granulosa cells and follicular fluid is remarkably higher in the PCOS group than the no-PCOS group. Additionally, the si- circ_0058063 group has a lower relative circ_0058063 expression, compared with the si-NC group in Fig. 1C. These outcomes reveal that the expression of circ_0058063 is elevated in PCOS ovarian granulosa cells and follicular fluid.

\subsection{Silencing circ_0058063 increases the levels of Aromatase mRNA, E2 and P4 in PCOS ovarian granulosa cells}

We use qRT-PCR to detect the Aromatase mRNA expression in ovarian granulosa cells. Additionally, ELISA assay is applied to assess P4 and E2 levels in ovarian granulosa cells. As can be seen from Fig. 2A-C, the levels of Aromatase mRNA, E2, and P4 are significantly increased in PCOS ovarian granulosa cells after silencing circ_0058063, compared with the si-NC group.

\subsection{Silencing circ_0058063 enhances cell proliferation in PCOS ovarian granulosa cells}

Cell proliferation is measured by CCK-8 assay. As demonstrated in Fig. 3A, the si-circ_0058063 group has remarkably higher ovarian granulosa cell proliferation than the si-NC group. In addition, western blot analysis is utilized to evaluate the Cyclin A and Cyclin D1 proliferation protein levels in each group. As displayed in Fig. 3B and 3C, the protein levels of Cyclin A and Cyclin D1 in the si- circ_0058063 group 


\subsection{Silencing circ_0058063 inhibits apoptosis of PCOS ovarian granulosa cells}

As presented in Fig. 4A and 4B, the results of flow cytometry suggest that cell apoptosis is significantly decreased after silencing circ_0058063 in PCOS ovarian granulosa cells, compared with the si-NC group. In Fig. 4C and 4D, western blot analysis indicates that silencing circ_0058063 significantly increases the expression of Bcl-2 protein and reduces the expression of Bax protein in the si-circ_0058063 group than in the si-NC group. These data show that silencing circ_0058063 inhibits apoptosis of PCOS ovarian granulosa cells.

\section{Discussion}

PCOS is the main cause of anovulatory infertility, which affects $80 \%$ of patients with anovulatory infertility $[12,13]$. At present, the mechanism of ovarian injury and abnormal follicular development in PCOS patients is not fully understood. PCOS is often closely related to repeated spontaneous abortions in infertile patients [14]. Therefore, we must pay attention to this group of women of childbearing age, who may suffer adverse consequences due to complications of PCOS.

Our study finds that the expression of circ_0058063 in ovarian granulosa cells and follicular fluid in the PCOS group is significantly up-regulated. Besides, silencing circ_0058063 increases the levels of Aromatase mRNA, E2, and P4 in PCOS ovarian granulosa cells. In addition, silencing circ_0058063 can promote the proliferation of ovarian granulosa cells in PCOS patients. Furthermore, the results of flow cytometry reveal that after silencing circ_0058063, the survival rate of PCOS ovarian granulocytes is significantly increased, and the apoptosis rate is significantly reduced, indicating that silencing circ_0058063 may play an inhibiting role in ovarian granulosa cell apoptosis and have a promoting effect on cell survival in PCOS.

Circ_0058063 is only been studied in esophageal squamous-cell carcinomas and bladder cancer $[10,15$, 16]. In the progression of bladder cancer, circRNA_0058063 serves as a ceRNA via sponging miR-4863p/FOXP4 and miR-145-5p/CDK6 axis [10, 16].Circ_0058063 stimulates glucose-uptake and increases the expression of GLUT1 in esophageal squamous-cell carcinomas [15]. To the best of our knowledge, the present study is the first to investigate the function of circ_0058063 in PCOS.

In conclusion, silencing circ_0058063 enhances proliferation and inhibits apoptosis in PCOS ovarian granulosa cells. Such findings may offer vital insights into a therapeutic target for PCOS.

\section{Declarations}




\section{Ethics approval and consent to participate}

This study was conducted after receiving approval from the Institutional Research Ethics Committee and the Research Ethics Committee of Huai'an Second People's Hospital, The Affiliated Huai'an Hospital of Xuzhou Medical University. Also, written informed consent was received from every patient who took part in the study.

\section{Consent for publication}

This is not relevant.

\section{Availability of data and material}

All materials and data of this study are available and can be requested from the corresponding author.

\section{Competing interests}

We declare that no conflicts of interest exist among authors or with the funding body.

\section{Funding}

This is not relevant.

\section{Authors' contributions}

Chengcai Wen and Li Zhang designed the experiments, conducted the experiments and wrote the manuscript. All of the authors gave final approval to the submitted version of the manuscript.

\section{Acknowledgements}

We thank all participants involved in this study.

\section{References}

1. Kelley CE, Brown AJ, Diehl AM, Setji TL. Review of nonalcoholic fatty liver disease in women with polycystic ovary syndrome. World J Gastroenterol. 2014; 20: 14172-84.

2. Hou Y, Wang Y, Xu S, Qi G, Wu X. Bioinformatics identification of microRNAs involved in polycystic ovary syndrome based on microarray data. Molecular medicine reports. 2019; 20: 281-91. 
3. Zhang R, Liu H, Bai H, Zhang Y, Liu Q, Guan L, Fan P. Oxidative stress status in Chinese women with different clinical phenotypes of polycystic ovary syndrome. Clin Endocrinol (Oxf). 2017; 86: 88-96.

4. Teede HJ, Misso ML, Deeks AA, Moran LJ, Stuckey BG, Wong JL, Norman RJ, Costello MF. Assessment and management of polycystic ovary syndrome: summary of an evidence-based guideline. The Medical journal of Australia. 2011; 195: S65-112.

5. Kaur R, Kaur T, Kaur A. Genetic association study from North India to analyze association of CYP19A1 and CYP17A1 with polycystic ovary syndrome. Journal of assisted reproduction and genetics. 2018; 35: 1123-9.

6. Meng X, Li X, Zhang P, Wang J, Zhou Y, Chen M. Circular RNA: an emerging key player in RNA world. Brief Bioinformatics. 2017; 18: 547-57.

7. Kristensen LS, Andersen MS, Stagsted LVW, Ebbesen KK, Hansen TB, Kjems J. The biogenesis, biology and characterization of circular RNAs. Nature reviews Genetics. 2019; 20: 675-91.

8. Ma Z, Zhao H, Zhang Y, Liu X, Hao C. Novel circular RNA expression in the cumulus cells of patients with polycystic ovary syndrome. Arch Gynecol Obstet. 2019; 299: 1715-25.

9. Zhang C, Liu J, Lai M, Li J, Zhan J, Wen Q, Ma H. Circular RNA expression profiling of granulosa cells in women of reproductive age with polycystic ovary syndrome. Arch Gynecol Obstet. 2019; 300: 43140.

10. Sun M, Zhao W, Chen Z, Li M, Li S, Wu B, Bu R. Circ_0058063 regulates CDK6 to promote bladder cancer progression by sponging miR-145-5p. J Cell Physiol. 2019; 234: 4812-24.

11. Revised 2003 consensus on diagnostic criteria and long-term health risks related to polycystic ovary syndrome (PCOS). Hum Reprod. 2004; 19: 41-7.

12. Balen AH, Morley LC, Misso M, Franks S, Legro RS, Wijeyaratne CN, Stener-Victorin E, Fauser BCJM, Norman RJ, Teede H. The management of anovulatory infertility in women with polycystic ovary syndrome: an analysis of the evidence to support the development of global WHO guidance. Hum Reprod Update. 2016; 22: 687-708.

13. Wang L, Qi H, Baker PN, Zhen Q, Zeng Q, Shi R, Tong C, Ge Q. Altered Circulating Inflammatory Cytokines Are Associated with Anovulatory Polycystic Ovary Syndrome (PCOS) Women Resistant to Clomiphene Citrate Treatment. Medical science monitor : international medical journal of experimental and clinical research. 2017; 23: 1083-9.

14. Zhang C, Liu J, Lai M, Li J, Zhan J, Wen Q, Ma H. Circular RNA expression profiling of granulosa cells in women of reproductive age with polycystic ovary syndrome. Arch Gynecol Obstet. 2019; 300: 43140.

15. Zheng Y, Chen Y, Jiang H, Zhang H, Wang H, Xu J, Yu Z. Circ_0058063 upregulates GLUT1 expression and promotes glucose-uptake in esophageal squamous-cell carcinomas. J Thorac Dis. 2020; 12: 925-31.

16. Liang H, Huang H, Li Y, Lu Y, Ye T. CircRNA_0058063 functions as a ceRNA in bladder cancer progression via targeting miR-486-3p/FOXP4 axis. Biosci Rep. 2020; 40. 
Figures

A

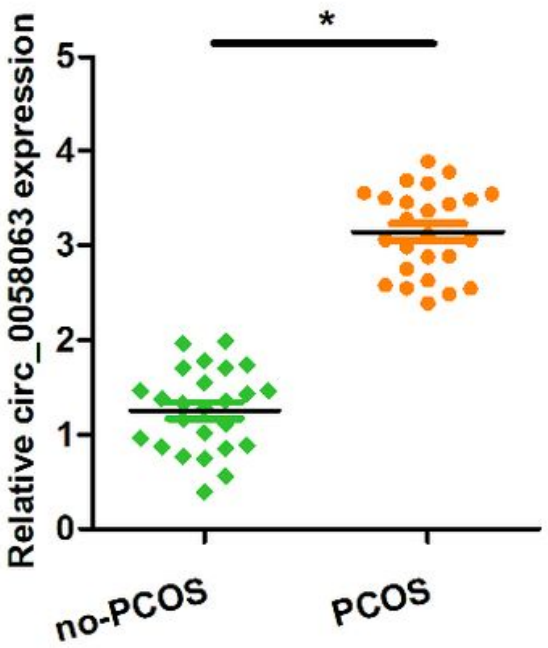

B

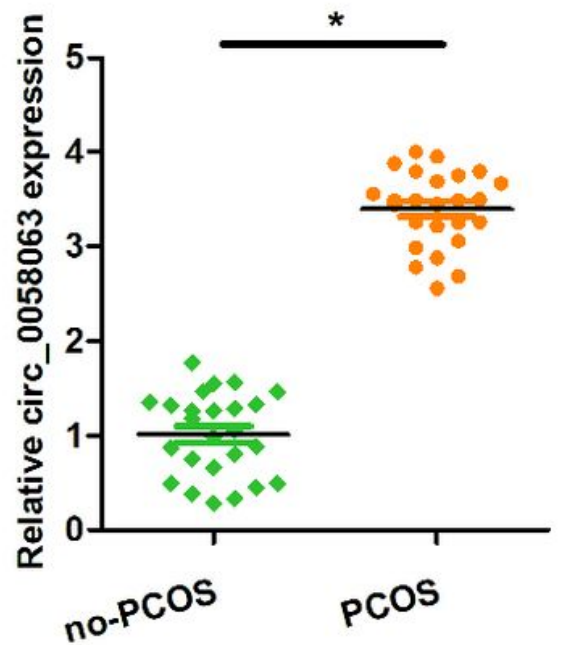

C

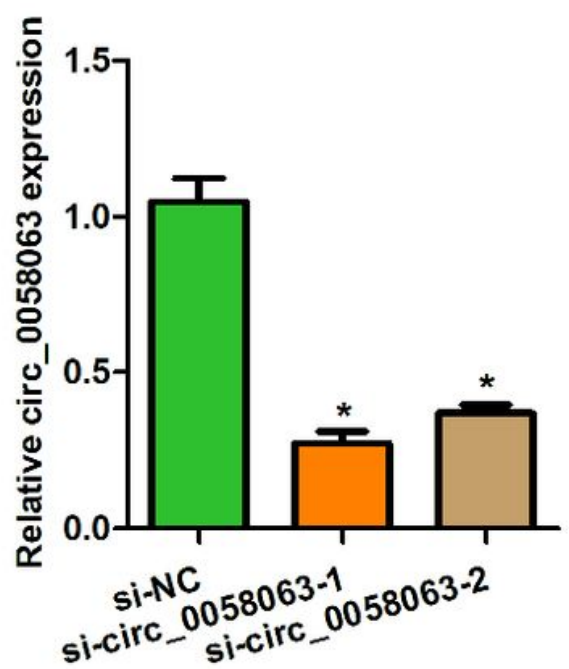

Figure 1

The expression of circ_0058063 is elevated in PCOS ovarian granulosa cells and follicular fluid (A) Expression of circ_0058063 in follicular fluid in two groups. (B) Relative circ_0058063 expression in ovarian granulosa cells in two groups. (C) Expression of circ_0058063 in ovarian granulosa cells in three groups. PCOS, polycystic ovary syndrome; NC, negative control. ${ }^{*}, \mathrm{P}<0.05$

A

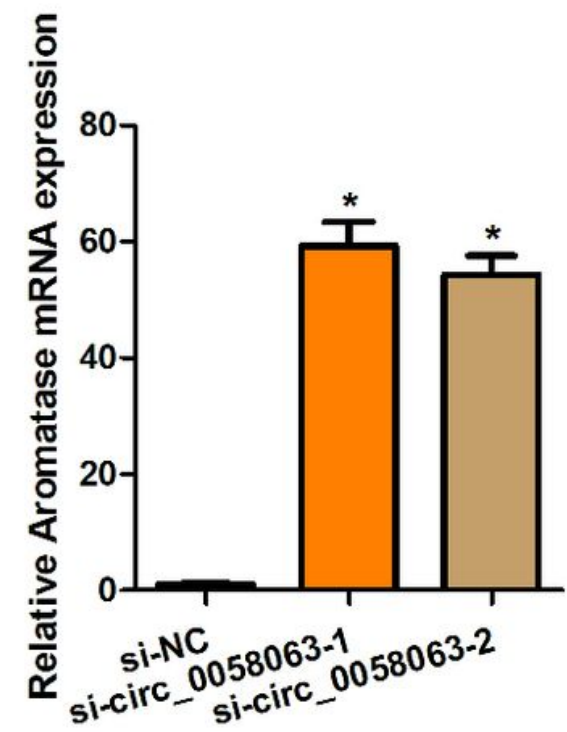

B

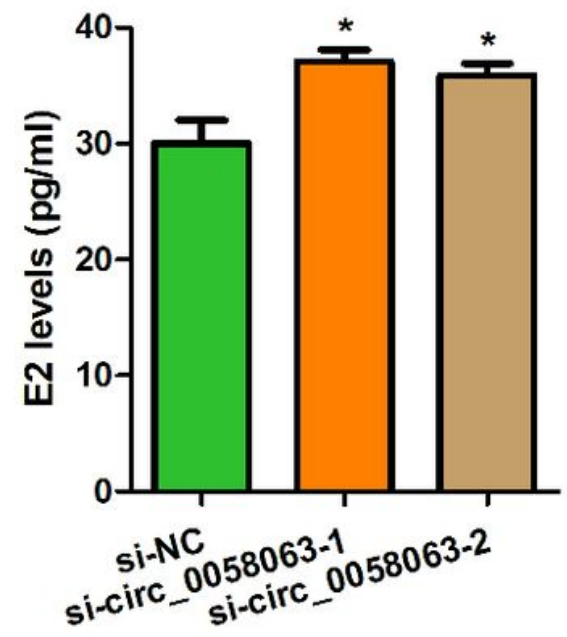

C

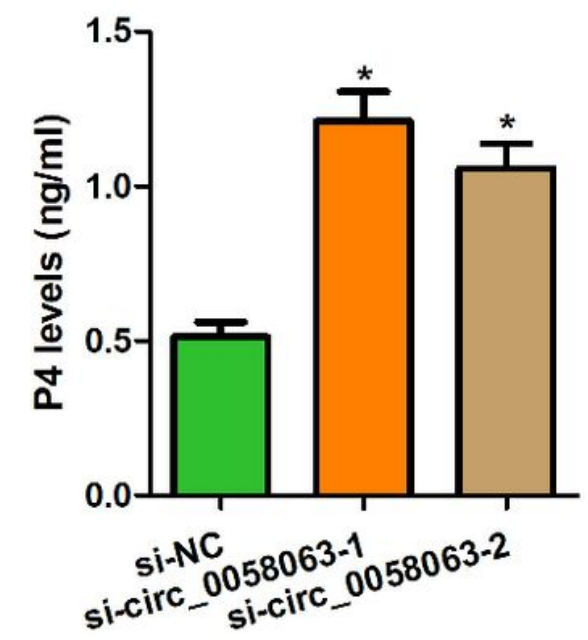

Figure 2 
Silencing circ_0058063 increases the levels of Aromatase mRNA, E2 and P4 in PCOS ovarian granulosa cells (A) Expression of Aromatase mRNA in ovarian granulosa cells in each group. (B) Levels of E2 in ovarian granulosa cells in each group. (C) Levels of P4 in ovarian granulosa cells in each group. PCOS, polycystic ovary syndrome; E2, estradiol; $\mathrm{P} 4$, progesterone; NC, negative control. ${ }^{*}, \mathrm{P}<0.05$

A

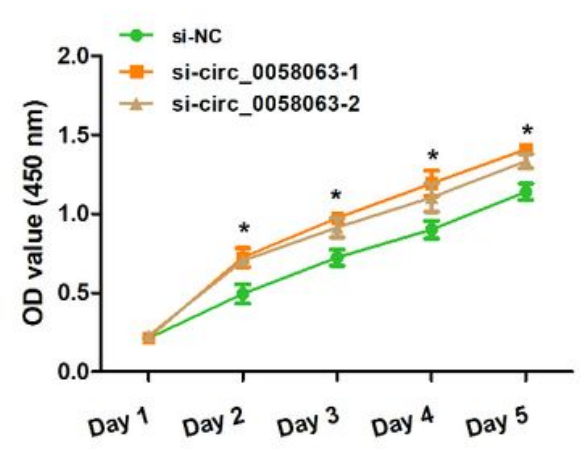

B

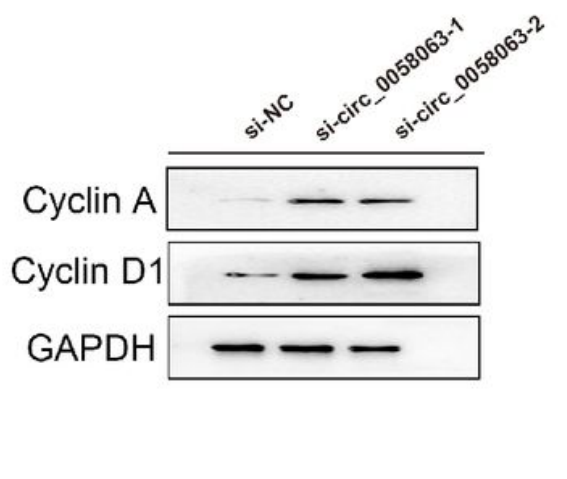

C

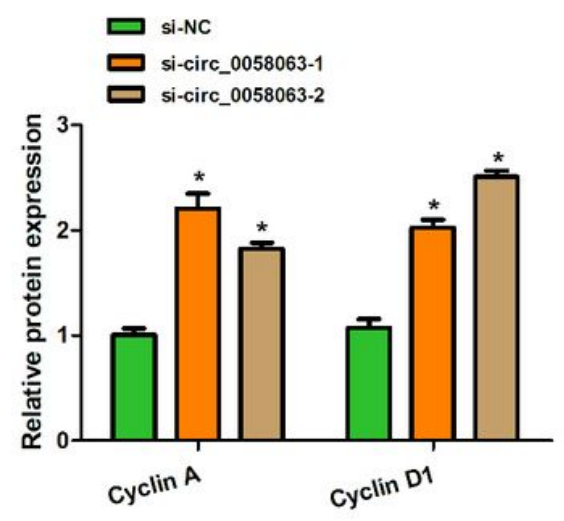

Figure 3

Silencing circ_0058063 enhances cell proliferation in PCOS ovarian granulosa cells (A) Cell proliferation of PCOS ovarian granulosa cells in each group. $(B, C)$ Relative protein expression of Cyclin $A$ and Cyclin D1 in each group. NC, negative control, *, $\mathrm{P}<0.05$

A

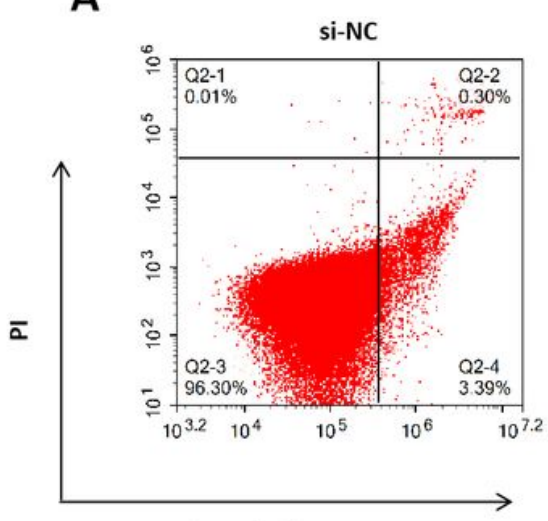

Annexin-V

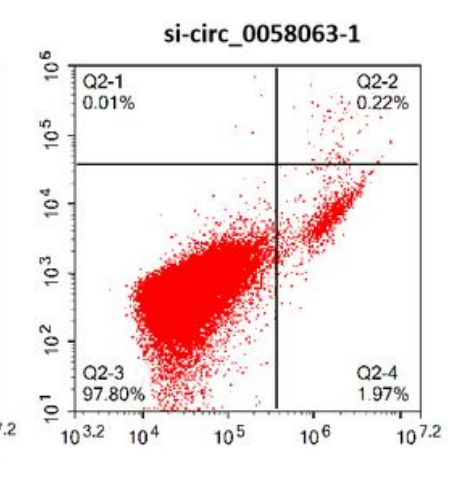

B
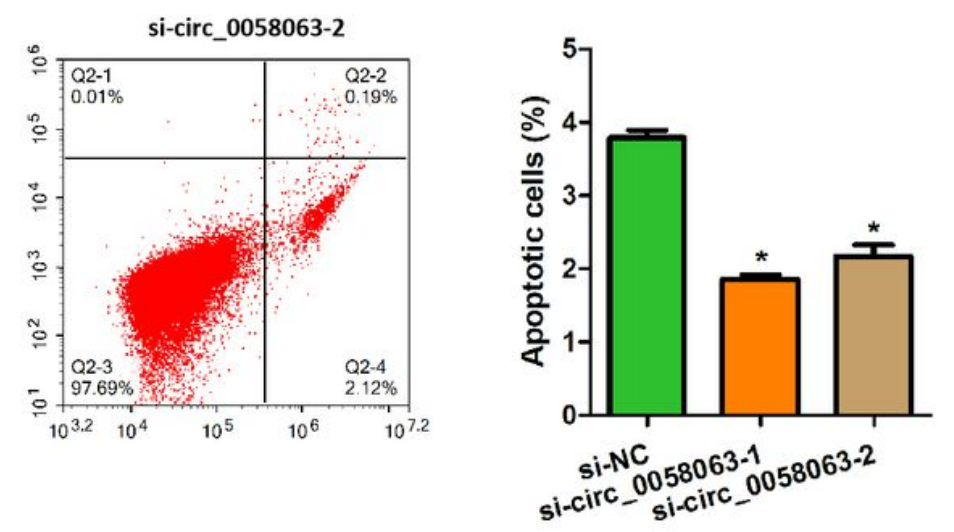

C

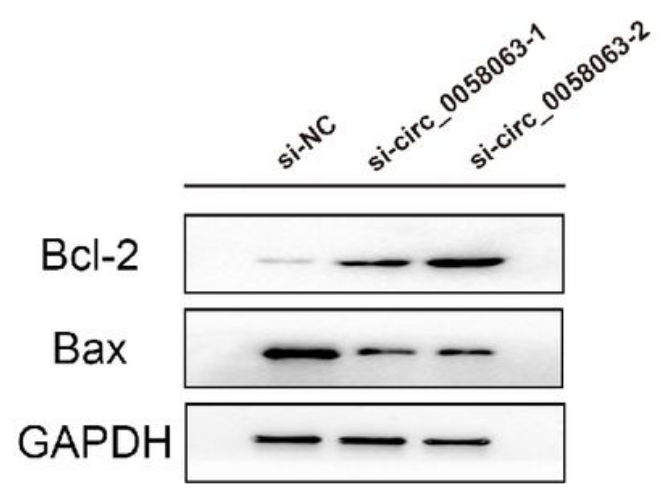

D

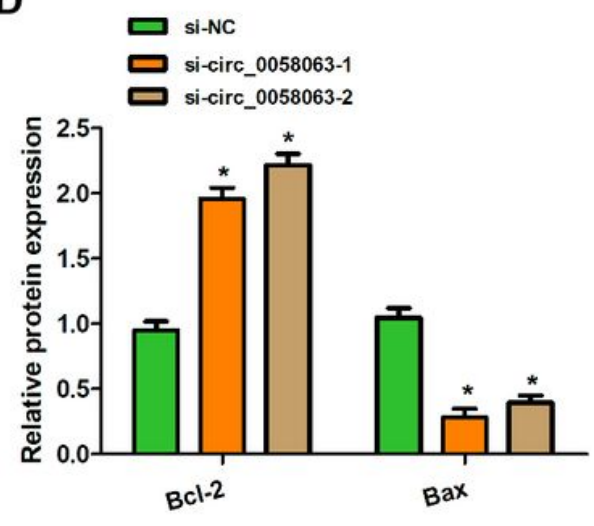




\section{Figure 4}

Silencing circ_0058063 inhibits apoptosis of PCOS ovarian granulosa cells (A, B) Cell apoptosis of PCOS ovarian granulosa cells was measured by flow cytometry assay; $(C, D) B C l-2$ and BAX protein expression in each group. NC, negative control, *, $\mathrm{P}<0.05$ 